\title{
$Q$-dependence of the inelastic neutron scattering cross section for molecular spin clusters with high molecular symmetry
}

\author{
O. Waldmann* \\ Physikalisches Institut III, Universität Erlangen-Nürnberg, D-91058 Erlangen, Germany
}

(Dated: September 6, 2018)

\begin{abstract}
For powder samples of polynuclear metal complexes the dependence of the inelastic neutron scattering intensity on the momentum transfer $Q$ is known to be described by a combination of so called interference terms. They reflect the interplay between the geometrical structure of the compound and the spatial properties of the wave functions involved in the transition. In this work, it is shown that the $Q$-dependence is strongly interrelated with the molecular symmetry of molecular nanomagnets, and, if the molecular symmetry is high enough, is actually completely determined by it. A general formalism connecting spatial symmetry and interference terms is developed. The arguments are detailed for cyclic spin clusters, as experimentally realized by e.g. the octanuclear molecular wheel $\mathrm{Cr}_{8}$, and the star like tetranuclear cluster $\mathrm{Fe}_{4}$.
\end{abstract}

\section{INTRODUCTION}

Molecular nanomagnets, inorganic complexes with tens of magnetic metal centers linked by organic ligands, are a new class of magnetic materials exhibiting fascinating quantum properties. For instance, quantum tunneling of the magnetization has been observed in clusters like $\mathrm{Mn}_{12}$ and $\mathrm{Fe}_{8}, \frac{1}{\underline{m}}$ now called single molecule magnets. Another class of molecular nanomagnets is established by the molecular wheels as represented by the prototype $\mathrm{Fe}_{10} \stackrel{2}{\stackrel{2}{2}}$ These antiferromagnetic cyclic clusters show pronounced steps in the magnetization curve at low temperature signaling quantum size effects ${ }^{2.3}$ Furthermore, the possibility of coherent tunneling of the Néel-vector has been suggested in these compounds. $\stackrel{4}{*}$ Yet other systems like molecular grids,,$\frac{5,6,7.8}{5}$ tetranuclear star like clusters,$\frac{9}{9}$ the cluster $\mathrm{V}_{15} \stackrel{10}{\underline{10}}$ or the magnetic Keplerate $\mathrm{Mo}_{72} \mathrm{Fe}_{30} \frac{11}{11}$ attracted much interest due to their peculiar magnetic properties.

The magnetism of these polynuclear complexes is, in principle, described by a microscopic spin Hamiltonian which to a very good first approximation consists of terms representing isotropic Heisenberg exchange interactions and dipole-dipole interactions among different spin centers within a molecule, and terms due to the ligand field and Zeeman interaction for each single ion ${ }^{12.13}$ Intermolecular interactions are usually very small and can be neglected. The isotropic Heisenberg terms are dominant in general and these clusters may be thus described as (antiferromagnetic) Heisenberg spin clusters with weak magnetic anisotropy.

For a detailed understanding of the properties of molecular nanomagnets it is of great importance to be able to study both the effects due to the Heisenberg interaction and anisotropic terms. Although rarely achieved in praxis for these rather large molecules, the ultimate goal would be to determine all relevant parameters of the microscopic spin Hamiltonian like exchange constants, single ion zero-field-splittings, etc.. Besides the obvious relevance in understanding the physics, the results are of great help also for synthetic chemists as they al- low to establish magneto-structural correlations which in turn provide ideas for a controlled improvement of these systems $\underline{12}$

Inelastic neutron scattering (INS) has been demonstrated to be a powerful experimental tool in this regard $\stackrel{14.15 .16 .17}{ }$ Being a spectroscopic technique, INS provides direct access to energy splittings. The INS cross section is controlled by the favorable selection rule $\Delta S=0, \pm 1$. INS thus allows to detect the splitting of individual spin multiplets, as $\operatorname{EPR}(\Delta S=0)$, but also the splitting produced by the magnetic interactions. As a result, INS provides a very straightforward determination of exchange parameters. It requires, however, large amounts of sample and, frequently, deuteration. Accordingly, only powder samples of molecular nanomagnets were investigated so far.

As a unique feature, INS additionally allows to measure the dependence of the INS intensity on the scattering vector $\mathbf{Q}$. In this work only the situation of powder samples will be considered, i.e. the INS cross section has to be averaged in $\mathbf{Q}$ space and the scattering intensity will depend only on the momentum transfer $Q \stackrel{14.15}{!}$ On the one hand, the $Q$-dependence allows to distinguish magnetic excitations from vibrational excitations $\frac{18}{18}$ On the other hand, and more importantly, the $Q$-dependence allows discriminating clearly between various types of magnetic transitions $\stackrel{14.15 .19}{ }$ Thus, the observed $Q$-dependence of a transition can be used in addition to its energy for a spectroscopic assignment removing ambiguities in many cases. This advantage has been explored in several works, a particular illustrative example is given by the tetranuclear cluster $\left[\mathrm{Co}_{4}\left(\mathrm{H}_{2} \mathrm{O}\right)_{2}\left(\mathrm{PW}_{9} \mathrm{O}_{34}\right)_{2}\right]$ for which the $Q$ dependence was key to establish the correct model ${ }^{20}$

As for the origin of the $Q$-dependence, it is well known that it depends sensitively on the wave functions of the states involved in a transition ${ }^{14.15}$ In the formula for the INS cross section the matrix elements for individual spin centers are correlated to their geometrical arrangement and the momentum transfer via a term $\exp \left[i \mathbf{Q}\left(\mathbf{R}_{i}-\mathbf{R}_{j}\right)\right]$. Here, $\mathbf{R}_{i}$ denotes the position vector of the $i$-th metal center. This gives rise to characteristic interference terms in the INS cross section for powder 
samples $^{21}$ This observation was expressed in Ref. 19. loosely as

where $\bar{I}(Q)$ denotes the averaged INS intensity and $F(Q)$ the magnetic form factor. It is thus clear, and well understood, that the $Q$-dependence basically senses the spatial properties of the involved wave functions. However, to the best of the authors knowledge, beyond this basic understanding the significance of Eq. (11) remained unexplored for larger spin systems like molecular nanomagnets.

For a limited number of small systems the $Q$-dependence could be calculated analytically, 14.19 .21 .22 .23 .24 thus detailing Eq. (11) for these cases. For larger, or more complex systems one has to resort to numerical procedures $20.25,26.27$ and a general program has been developed $\stackrel{28}{ }$ However, the "black box" character of this approach did not yield insight into the relation for large spin systems between geometrical structure and wave functions as expressed by the interference terms.

This work aims at showing how the $Q$-dependence is influenced by the molecular symmetry of the spin cluster and in particular, if the molecular symmetry is high enough, that it is actually predetermined by it. The arguments will be detailed for the particular cases of the cyclic spin clusters with focus on the molecular wheel $\mathrm{Cr}_{8} \stackrel{29}{\underline{29}}$ and the tetranuclear star like cluster $\mathrm{Fe}_{4} \stackrel{\underline{\underline{9}}}{\text { The }}$ molecular wheel $\mathrm{Cr}_{8}$ and star like cluster $\mathrm{Fe}_{4}$ were chosen as examples as they both exhibit a very high molecular symmetry and detailed INS experiments were performed recently 30.31 .32 Additionally, the Heisenberg interaction is much stronger than the anisotropic terms in both compounds. INS measurements on powder samples thus provide an excellent view on their internal spin structure. Several transitions between different spin multiplets were observed experimentally and only these are of interest here. As a main result it will be demonstrated that for such highly symmetric systems the $Q$-dependence actually can be used to determine experimentally the spatial quantum numbers of the states involved. The molecular wheel $\mathrm{Cr}_{8}$ is a particularly illustrative example in this respect.

In the next section, first the general equation for the INS cross section of powder samples will be derived, correcting an earlier result ${ }^{28}$ Then, in section IIIthe general framework based on standard group theoretical procedures will be developed. In sections IV and $\nabla$ the molecular wheel $\mathrm{Cr}_{8}$ and the star like cluster $\mathrm{Fe}_{4}$ will be discussed. The work concludes with section VI

\section{INS CROSS SECTION FOR POWDER SAMPLES}

The differential neutron scattering cross section is ${ }^{14,15}$

$$
\frac{d^{2} \sigma}{d \Omega d \omega}=C(Q, T) \sum_{n m} \frac{e^{-\beta E_{n}}}{Z(T)} I_{n m}(\mathbf{Q}) \delta\left(\omega-\frac{E_{m}-E_{n}}{\hbar}\right)
$$

where $C(Q, T)=\left(\gamma e^{2} / m_{e} c^{2}\right)\left(k^{\prime} / k\right) \exp [-2 W(Q, T)]$, $\beta=1 /\left(k_{B} T\right), Z(T)$ the partition function, and

$$
\begin{array}{r}
I_{n m}(\mathbf{Q})=\sum_{i j} F_{i}^{*}(Q) F_{j}(Q) e^{i \mathbf{Q} \cdot \mathbf{R}_{i j}} \times \\
\sum_{\alpha \beta}\left(\delta_{\alpha \beta}-\frac{Q_{\alpha} Q_{\beta}}{Q^{2}}\right)\left\langle n\left|S_{i \alpha}\right| m\right\rangle\left\langle m\left|S_{j \beta}\right| n\right\rangle .
\end{array}
$$

In this equation, $F_{i}(Q)$ is the magnetic form factor of the $i$-th spin center, $\alpha, \beta=x, y, z, \mathbf{Q}=\mathbf{k}^{\prime}-\mathbf{k}$ is the transferred momentum, and $\mathbf{R}_{i j}=\mathbf{R}_{i}-\mathbf{R}_{j}$ with the spin position vectors $\mathbf{R}_{i}$. In the following, the abbreviations $l_{\alpha}=Q_{\alpha} / Q$ and $\tilde{S}_{i \alpha}$ for the matrix elements of $S_{i \alpha}$ are used. In an ordered product $\tilde{S}_{i \alpha} \tilde{S}_{j \beta}$, the first term always has to be understood as a matrix element between $\langle n|$ and $|m\rangle$ and the second as between $\langle m|$ and $|n\rangle$.

The cross section for a powder sample is obtained by averaging Eq. (2) over all directions of $\mathbf{Q}$, i.e. one has to calculate the expression

$$
\bar{I}_{i j, n m}(Q)=\int \frac{d \Omega}{4 \pi} e^{i \mathbf{Q} \cdot \mathbf{R}_{i j}} \sum_{\alpha \beta}\left(\delta_{\alpha \beta}-l_{\alpha} l_{\beta}\right) \tilde{S}_{i \alpha} \tilde{S}_{j \beta} .
$$

This is conveniently done by resorting to the calculus of irreducible spherical tensors 33 The sum over $\alpha$ and $\beta$ can be rearranged to $\sum_{\alpha} \tilde{S}_{i \alpha} \tilde{S}_{j \alpha}-\left(\sum_{\alpha} l_{\alpha} \tilde{S}_{i \alpha}\right)\left(\sum_{\beta} l_{\beta} \tilde{S}_{j \beta}\right)$. The first term in this expression is easily averaged since it does not depend on the orientation of $\mathbf{Q}$, yielding $j_{0}\left(Q R_{i j}\right) \tilde{\mathbf{S}}_{i} \cdot \tilde{\mathbf{S}}_{j}$. Here, $j_{k}$ is the spherical Bessel function of order $k$. The second term is calculated by expressing the scalar product $\sum_{\alpha} l_{\alpha} \tilde{S}_{i \alpha}$ by spherical tensors ${ }^{33}$ according to

$$
\sum_{\alpha} l_{\alpha} \tilde{S}_{i \alpha}=\sum_{q} T_{q}^{(1) *}(\mathbf{Q}) T_{q}^{(1)}\left(\tilde{\mathbf{S}}_{i}\right)
$$

$T_{q}^{(k)}(\mathbf{O})$ denotes the $q$-th component of the irreducible spherical tensor of degree $k$ with respect to the vector $\mathbf{O}$. They are proportional to the spherical harmonics $Y_{q}^{k}(\mathbf{O})$. Expanding the exponential in spherical harmonics and using the coupling rule for spherical tensors one obtains

$$
\bar{I}_{i j, n m}(Q)=\sqrt{4 \pi} \sum_{L=0,2} i^{L} j_{L}\left(Q R_{i j}\right)\left(\begin{array}{ccc}
1 & 1 & L \\
0 & 0 & 0
\end{array}\right) \times
$$




$$
\sum_{M} Y_{M}^{L *}\left(\mathbf{R}_{i j}\right)\left[T^{(1)}\left(\tilde{\mathbf{S}}_{i}\right) \otimes T^{(1)}\left(\tilde{\mathbf{S}}_{j}\right)\right]_{M}^{(L)} .
$$

Here, (...) denotes a Wigner-3 $j$ symbol. The term for $L=0$ gives $\frac{1}{3} j_{0}\left(Q R_{i j}\right) \tilde{\mathbf{S}}_{i} \cdot \tilde{\mathbf{S}}_{j}$. For $\bar{I}_{n m}(Q)=$ $\int d \Omega / 4 \pi I_{n m}(\mathbf{Q})$ one finally obtains

$$
\begin{array}{r}
\bar{I}_{n m}(Q)=\sum_{i j} F_{i}^{*}(Q) F_{j}(Q)\left(\frac{2}{3} j_{0}\left(Q R_{i j}\right) \tilde{\mathbf{S}}_{i} \cdot \tilde{\mathbf{S}}_{j}+\right. \\
\left.j_{2}\left(Q R_{i j}\right) \sum_{M} T_{M}^{(2) *}\left(\mathbf{R}_{i j}\right)\left[T^{(1)}\left(\tilde{\mathbf{S}}_{i}\right) \otimes T^{(1)}\left(\tilde{\mathbf{S}}_{j}\right)\right]_{M}^{(2)}\right) .
\end{array}
$$

The $L=2$ term could be put also into the more compact form $j_{2}\left(Q R_{i j}\right) \mathbf{T}^{(2)}\left(\mathbf{R}_{i j}\right) \cdot \mathbf{T}^{(2)}\left(\tilde{\mathbf{S}}_{i} \tilde{\mathbf{S}}_{j}\right)$ using Eq. (5).

Several special cases shall be discussed. It is interesting to consider the limit $Q \rightarrow 0$. Since $j_{0}(x \rightarrow 0) \approx 1+\mathcal{O}\left(x^{2}\right)$ and $j_{2}(x \rightarrow 0) \approx x^{2} / 5$, one simply obtains

$$
\bar{I}_{n m}(Q \rightarrow 0)=\frac{2}{3} \sum_{i j} F_{i}^{*}(Q) F_{j}(Q) \tilde{\mathbf{S}}_{i} \cdot \tilde{\mathbf{S}}_{j}+\mathcal{O}\left(Q^{2}\right) .
$$

Next, for an isotropic spin cluster the $L=2$ term in Eq. (7) obviously vanishes. If one further numbers the different possible values of $R_{i j}$ by $p$ and writes $R_{p}$, the result assumes the convenient form

$$
\bar{I}_{n m}^{i s o}(Q, T)=\sum_{p} I_{n m}^{p}(Q, T) j_{0}\left(Q R_{p}\right) .
$$

Accordingly, the scattering intensity is sort of an expansion in $j_{0}\left(Q R_{p}\right)$. In principle, since the $R_{p}$ are known, the coefficients $I_{p}$ can be determined by fitting the $Q$ dependence of the scattering intensity to Eq. (9). Equation (9) precises Eq. (1) for the case of isotropic systems.

Finally, one may consider the case where only the components with $\alpha=\beta$ contribute. This holds if $\left(\tilde{S}_{i, \alpha} \tilde{S}_{j \beta}+\tilde{S}_{i \beta} \tilde{S}_{j \alpha}\right)=0$ for all $\alpha \neq \beta$. However, it is clear that this condition is not fulfilled in general. One necessary condition is that the quantization axis for the spin operators coincide with a magnetic main axis. For uniaxial and isotropic systems this is sufficient to ensure the above condition. However, for a biaxial magnetic system this is not sufficient in general since in the neutron scattering cross section the local terms $\tilde{S}_{i \alpha} \tilde{S}_{j \beta}$ enter while "biaxial" is a property of the whole spin cluster as an entity. Thus, for spin systems with magnetic symmetry lower than uniaxial the validity of this simplification has to be checked carefully. For an uniaxial system only the $M=0$ term contributes, resulting in

$$
\begin{gathered}
\bar{I}_{n m}^{u n i}(Q)=\frac{2}{3} \sum_{i j} F_{i}^{*}(Q) F_{j}(Q) \times \\
\left\{\left[j_{0}\left(Q R_{i j}\right)-\frac{1}{2} j_{2}\left(Q R_{i j}\right) C_{0}^{2}\left(\mathbf{R}_{i j}\right)\right]\left(\tilde{S}_{i x} \tilde{S}_{j x}+\tilde{S}_{i y} \tilde{S}_{j y}\right)\right. \\
\left.+\left[j_{0}\left(Q R_{i j}\right)+j_{2}\left(Q R_{i j}\right) C_{0}^{2}\left(\mathbf{R}_{i j}\right)\right] \tilde{S}_{i z} \tilde{S}_{j z}\right\}(10)
\end{gathered}
$$

\section{INS CROSS SECTION AND SPATIAL SYMMETRY}

The spatial symmetry of the molecule leads to an invariance of the spin Hamiltonian upon certain permutations of the spin centers $\stackrel{34.35}{ }$ The group of all these permutations is denoted as $\mathcal{G}$, and an element of this group as $P$. It should be noted that $\mathcal{G}$ and the point group of the molecule are not equivalent in general. This symmetry of the spin Hamiltonian is accordingly denoted here as spin permutational symmetry ${ }^{35}$ Its effects are exploited by applying the standard results of group theory 33.34

The eigenstates $|n\rangle$ of the spin Hamiltonian can be classified by the irreducible representations (IRs) of $\mathcal{G}$ and are written now as $|\tau k \mu\rangle$. They transform according to $O(P)|\tau k \mu\rangle=\sum_{\bar{\mu}} \Gamma_{\bar{\mu} \mu}^{(k)}(P)|\tau k \bar{\mu}\rangle . O(P)$ denotes the operator associated with the permutation $P$. Furthermore, $O(P) S_{i \alpha} O\left(P^{-1}\right)=\Gamma_{j i}(P) S_{j \alpha}$. Here, no sum over $j$ arises on the r.h.s since $P$ is a permutation, i.e. $P i \neq P j$ for $i \neq j$. Accordingly, each row or column, respectively, of the matrix $\boldsymbol{\Gamma}(P)$ has only exactly one nonzero entry which is equal to 1 . With these relationships one confirms

$$
\begin{array}{r}
\left\langle\tau^{\prime} k^{\prime} \mu^{\prime}\left|S_{j \beta}\right| \tau k \mu\right\rangle= \\
\sum_{\bar{\mu}^{\prime} \bar{\mu}}\left\langle\tau^{\prime} k^{\prime} \bar{\mu}^{\prime}\left|S_{i \beta}\right| \tau k \bar{\mu}\right\rangle \Gamma_{\bar{\mu}^{\prime} \mu^{\prime}}^{\left(k^{\prime}\right) *}\left(P_{j i}\right) \Gamma_{\bar{\mu} \mu}^{(k)}\left(P_{j i}\right)
\end{array}
$$

where $P_{j i}$ is a symmetry element which maps the $j$ th spin exactly onto the $i$-th spin, i.e. $P_{j i} j=i$. Equation (II) describes the restrictions imposed by the molecular symmetry on the interference effects in inelastic neutron scattering, and is thus central to this work.

The spin centers can be divided into classes such that all the spin centers of one class are related by the permutations of the group $\mathcal{G}$. More precisely, a class is a set of spin centers which transform one into another under the operations of $\mathcal{G}$. The different classes will be numbered by $\gamma$. By construction, each spin center is member of exactly one class. Therefore,

$$
\sum_{i}=\sum_{\gamma} \sum_{i \in \gamma}
$$

From each class, one spin center is chosen (arbitrarily) and called the pivotal center of this class. It will be also indicated by $\gamma$. Equation (11) then essentially provides a relation between the matrix element of an arbitrary spin center with the matrix element of its pivotal center. This imposes a certain structure on the expression $\sum_{i j} F_{i}^{*}(Q) F_{j}(Q) f\left(\mathbf{Q}, \mathbf{R}_{i j}\right) \tilde{S}_{i \alpha} \tilde{S}_{j \beta}$, which, with $f\left(\mathbf{Q}, \mathbf{R}_{i j}\right)$ appropriately chosen, is the part of the cross section relevant here. This idea can be used to work out general expressions based on the generalized WignerEckart theorem ${ }^{33}$

For spin permutational groups with only onedimensional IRs, writing the eigen states as $|\tau k\rangle$, Eq. (11) 
simplifies to $\left\langle\tau^{\prime} k^{\prime}\left|S_{j \beta}\right| \tau k\right\rangle=\left\langle\tau^{\prime} k^{\prime}\left|S_{i \beta}\right| \tau k\right\rangle \hat{\chi}^{k^{\prime} k}\left(P_{j i}\right)$. Whereby, $\hat{\chi}^{k k^{\prime}}(P)=\chi^{k *}(P) \chi^{k^{\prime}}(P)$ and $\chi^{k}$ being the character of the $k$-th IR. Together with Eq. (12) and after some rearrangement of terms one obtains the central result of this work:

$$
\begin{array}{r}
\sum_{i j} F_{i}^{*}(Q) F_{j}(Q) f\left(\mathbf{Q}, \mathbf{R}_{i j}\right) \tilde{S}_{i \alpha} \tilde{S}_{j \beta}= \\
\sum_{\gamma \bar{\gamma}} \tilde{S}_{\gamma \alpha} \tilde{S}_{\bar{\gamma} \beta} F_{\gamma}^{*}(Q) F_{\bar{\gamma}}(Q) f_{\gamma \bar{\gamma}}^{k k^{\prime}}(\mathbf{Q}) \\
f_{\gamma \bar{\gamma}}^{k k^{\prime}}(\mathbf{Q})=\sum_{i \in \gamma} \sum_{j \in \bar{\gamma}} f\left(\mathbf{Q}, \mathbf{R}_{i j}\right) \hat{\chi}^{k k^{\prime}}\left(P_{i \gamma}\right) \hat{\chi}^{k^{\prime} k}\left(P_{j \bar{\gamma}}\right) .
\end{array}
$$

The $Q$-dependence of the neutron scattering cross section is given by the interference terms $f_{\gamma \bar{\gamma}}^{k k^{\prime}}(\mathbf{Q})$ which can be calculated without knowledge of the wave functions, i.e. are completely governed by the spatial symmetry properties. These equations, being a precise statement of Eq. (1), become the more useful the smaller the number of classes $\gamma$ produced by the spin permutational symmetry is.

\section{CYCLIC SPIN CLUSTERS}

The full spin permutational symmetry group of a cyclic spin cluster is $D_{N}$, where $N$ is the number of spin centers. However, the spin levels may be also classified by the IRs of the subgroup $C_{N}$ reflecting the translational invariance $\stackrel{34}{=}$ This effectively introduces the shift quantum number $q=0, \ldots, N-1$ via the shift operator $T$ : $T|\tau q\rangle=e^{-i q 2 \pi / N}|\tau q\rangle$. The group elements may be written as $T^{n}$ with $n=0, \ldots, N-1$ and the characters are $\chi^{(q)}\left(T^{n}\right)=e^{-i q n 2 \pi / N}$. The full symmetry group $D_{N}$ has one-dimensional and two-dimensional IRs. Spin levels with $q=0$ and $q=N / 2$ belong to one-dimensional IRs of $D_{N}$, while for $q \neq 0, N / 2$ the two states with $q$ and $N-q$ belong to a two-dimensional IR. The latter are thus degenerate.

The interference terms $f_{\gamma \bar{\gamma}}^{k k^{\prime}}(\mathbf{Q})$ can now be calculated. For each spin center there is always one symmetry element $T^{n}$ which connects it to any other spin center. Thus, there is only one class $\gamma: \gamma=\{1, \ldots, N\}$. Spin center 1 is chosen as pivotal center. For $P_{i \gamma}$ one establishes that $P_{i \gamma}=P_{i 1}=T^{i-1}$, and accordingly that $\hat{\chi}^{q q^{\prime}}\left(P_{i \gamma}\right) \hat{\chi}^{q^{\prime} q}\left(P_{j \gamma}\right)=e^{i\left(q-q^{\prime}\right)(j-i) 2 \pi / N}$. Introducing the "distance" $n$ by $j=i+n$ yields

$$
f^{q q^{\prime}}(\mathbf{Q})=\sum_{n=0}^{N-1} \sum_{i=1}^{N} f\left(\mathbf{Q}, \mathbf{R}_{i, i+n}\right) e^{i \frac{2 \pi}{N}\left(q-q^{\prime}\right) n} .
$$

The cyclic spin clusters synthesized so far exhibit at best a molecular $S_{N}$ symmetry axis, as determined by x-ray crystallography ${ }^{36.37 .38}$ The magnetic anisotropy of these systems is thus expected to be strictly uniaxial, as is supported by experiments $\frac{39.40}{2}$ The position vectors $\mathbf{R}_{i}$ point to above and below the plane of the molecule perpendicular to the main symmetry axis. However, even with this effect $f\left(\mathbf{Q}, \mathbf{R}_{i, i+n}\right)$ becomes independent on $i$ for powder samples, i.e. $R_{i, i+n} \rightarrow R_{n}$ and $C_{0}^{2}\left(\mathbf{R}_{i, i+n}\right) \rightarrow C_{0}^{2}\left(\mathbf{R}_{n}\right)$ [see Eq. (10)]. Thus, for powder samples of these highly symmetric, cyclic spin clusters the interference terms are given by

$$
f^{q q^{\prime}}(\mathbf{Q})=N \sum_{n=0}^{N-1} f\left(\mathbf{Q}, \mathbf{R}_{n}\right) e^{i \frac{2 \pi}{N}\left(q-q^{\prime}\right) n} .
$$

The averaged INS cross section for the transition $|\tau q\rangle \rightarrow\left|\tau^{\prime} q^{\prime}\right\rangle$ is then simply proportional to

$$
\begin{gathered}
\bar{I}_{\tau q \tau^{\prime} q^{\prime}}(Q)=\frac{2}{3} N F^{2}(Q)\left\{\tilde{\mathbf{S}}_{1} \cdot \tilde{\mathbf{S}}_{1} f_{q q^{\prime}}^{0}(Q)+\right. \\
\left.\frac{1}{2}\left(2 \tilde{S}_{1 z} \tilde{S}_{1 z}-\tilde{S}_{1 x} \tilde{S}_{1 x}-\tilde{S}_{1 y} \tilde{S}_{1 y}\right) f_{q q^{\prime}}^{2}(Q)\right\}
\end{gathered}
$$

with

$$
\begin{aligned}
& f_{q q^{\prime}}^{0}(Q)=\sum_{n=0}^{N-1} j_{0}\left(Q R_{n}\right) e^{i \frac{2 \pi}{N}\left(q-q^{\prime}\right) n} \\
& f_{q q^{\prime}}^{2}(Q)=\sum_{n=0}^{N-1} j_{2}\left(Q R_{n}\right) C_{0}^{2}\left(\mathbf{R}_{n}\right) e^{i \frac{2 \pi}{N}\left(q-q^{\prime}\right) n}
\end{aligned}
$$

Apart from two normalizing factors, the $Q$-dependence of the INS intensity of uniaxial cyclic clusters is analytically determined by their spatial symmetry properties. The interference terms $f_{q q^{\prime}}^{0}(Q)$ and $f_{q q^{\prime}}^{2}(Q)$ do not depend on $\tau$ and $\tau^{\prime}$; they are completely determined by the spatial quantum numbers.

The following general result is noteworthy. In the limit $Q \rightarrow 0$, Eq. (16) leads to

$$
\begin{aligned}
\bar{I}_{\tau q \tau^{\prime} q^{\prime}}(Q) & =\frac{2}{3} N F^{2}(Q) \tilde{\mathbf{S}}_{1}^{2} \sum_{n=0}^{N-1} e^{i \frac{2 \pi}{N}\left(q-q^{\prime}\right) n}+\mathcal{O}\left(Q^{2}\right) \\
& \propto \delta_{q q^{\prime}}+\mathcal{O}\left(Q^{2}\right) .
\end{aligned}
$$

Therefore, comparing with Eq. (8), the spatial symmetry of the cyclic spin cluster enforces the INS intensity to become strictly zero for $Q=0$ if $q \neq q^{\prime}$, i.e. if the spatial quantum numbers of the states involved are different. In this case, the intensity actually approaches zero as $I(Q) \propto Q^{2}$. Equation (17) might have important experimental implications. Recently it became an intensively discussed question whether cyclic clusters like the ferric wheels are accurately described by a spin Hamiltonian with perfect cyclic symmetry, or whether additional terms with less symmetry are of importance ${ }^{41,42}$ In principle, Eq. (17) provides a powerful experimental approach to unravel this question since if symmetry breaking terms are relevant the INS intensity would not drop to zero with $Q \rightarrow 0$. 
Eq. (16) shall be worked out explicitly for the case of an octanuclear ring with predominantly antiferromagnetic next neighbor interactions, as appropriate for the so called $\mathrm{Cr}_{8}$ cluster ${ }^{29}$ This is motivated by recent, very detailed and accurate INS measurements on this system ${ }^{32}$ The $Q$-dependence of the INS intensity has been measured earlier also on a hexanuclear iron(III) cluster, but has been found afterwards to be less reliable due to instrumental artifacts $\underline{39.43}$

For an octanuclear ring with molecular $S_{4}$ symmetry, the position vectors are simply $\mathbf{R}_{i}=$ $R_{0}\left(\cos (i \pi / 4), \sin (i \pi / 4),(-1)^{i} R_{z}\right)$, where $R_{0}$ is the "radius" of the ring $\left(R_{0}=4.427 \AA\right.$ for $\left.\mathrm{Cr}_{8}\right)$ and $R_{z}$ determines by how much the ions lie above or below the plane of the ring. Since $R_{z} \ll R_{0}$, it shall be neglected here (but can be incorporated straightforwardly if felt to be required). For $R_{n}$ then holds $R_{1}=R_{7}=\sqrt{2-\sqrt{2}} R_{0}$, $R_{2}=R_{6}=\sqrt{2} R_{0}, R_{3}=R_{5}=\sqrt{2+\sqrt{2}} R_{0}$, and $R_{4}=2 R_{0}$. Also, it follows that $C_{0}^{2}\left(\mathbf{R}_{n}\right)=-1 / 2$. This is actually valid for the weaker condition $R_{z}^{2} \ll R_{0}^{2}$.

For finite rings with antiferromagnetic Heisenberg interactions it is well established that, as function of the total spin quantum number $S$, the lowest lying states form a set of rotational bands ${ }^{44}$ The energies of the states of a particular rotational band follow the Landé rule $E(S) \propto S(S+1)$ as for a rigid rotator 44.45 The set of bands can be distinguished into $L$ - and $E$-band: The $L$-band consist of the states for which $q$ toggles as function of $S$ between $q=0$ and $q=N / 2$, the $E$-band embraces the lowest lying states with $q \neq 0, N / 2$, which are essentially spin waves in character $\stackrel{44.46}{ }$ The energies of the states of these bands can be approximated excellently by $E(S, q)=\frac{1}{2} \Delta S(S+1)+\epsilon(q)$. $\Delta$ characterizes the "curvature" of the bands and coincides with the energy gap between ground and first excited state. $\epsilon(q)$ measures the "offset" of a particular band, as classified by $q$. Whereby, $\epsilon(q) \approx$ const $|\sin (2 \pi q / N)| \stackrel{44.46}{\stackrel{4}{*} \text { Impor- }}$ tantly, the $L$-band is well separated in energy from the higher lying $E$-bands, i.e. $\Delta \ll \epsilon(q)$ for $q \neq 0, N / 2$.

In actual INS experiments only the transitions between states within the $L$-band and those from the $L$-band into the $E$-band are relevant since $\Delta$ is typically on the order of meV. For transitions within the $L$-band $q-q^{\prime}=N / 2$ holds; and for transitions from the $L$-band into the $E$ band $q-q^{\prime} \neq 0, N / 2$ (values for $q$ should always be understood modulo $N)$.

First, the $L=0$ contribution in Eq. (16), i.e. $f_{q q^{\prime}}^{0}(Q)$ will be considered. For $\mathrm{Cr}_{8}, q-q^{\prime}=4$ for transitions between states of the $L$-band, directly yielding

$$
f_{0,4}^{0}=1+2 \sum_{n=1}^{3}(-1)^{n} j_{0}\left(Q R_{n}\right)+j_{0}\left(Q R_{4}\right)
$$

for the interference term. For transitions from a state of the $L$-band to states of the $E$-band one additionally has to take into account the degeneracy of the states with $q$ and $N-q$ due to the overall $D_{N}$ symmetry. However,
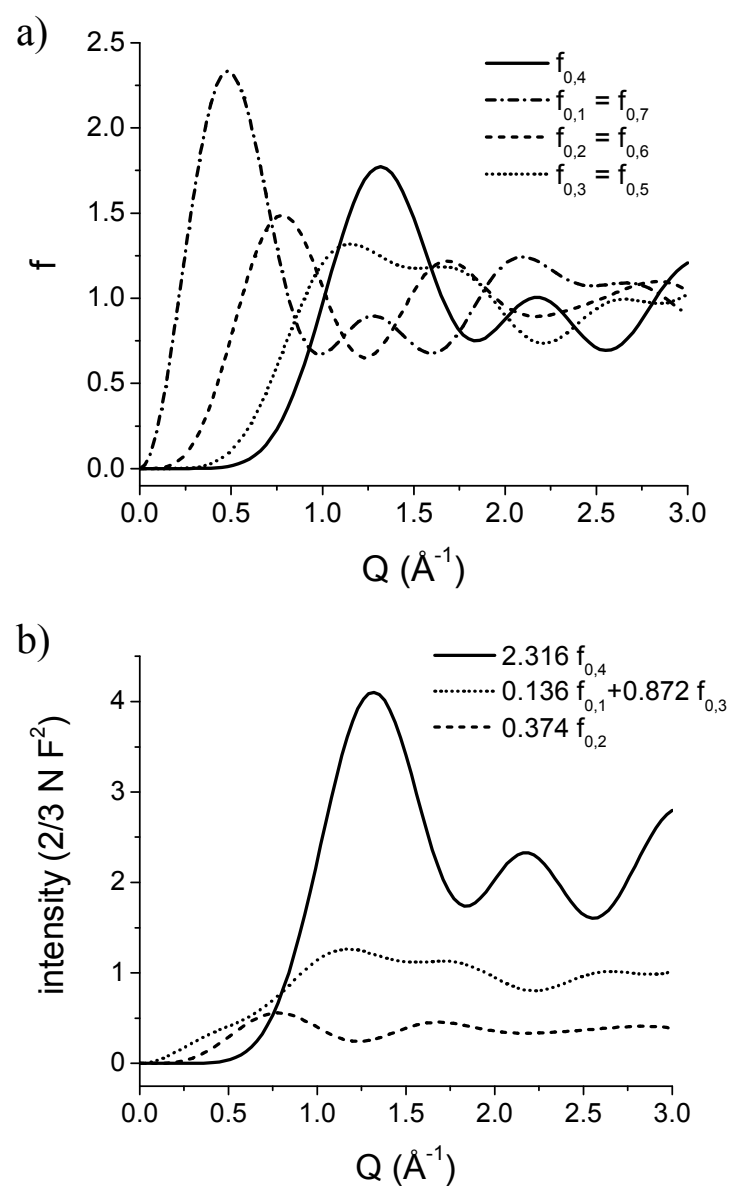

FIG. 1: (a) $Q$-dependence of the interference terms $f_{0,4}^{0}, f_{0,1}^{0}$, $f_{0,2}^{0}$ and $f_{0,3}^{0}$ as given in Eq. (18) for an octanuclear cyclic ring. (b) Dependence of the inelastic neutron scattering intensity as function of momentum transfer $Q$ for the three observable transitions at low temperatures of an octanuclear antiferromagnetic Heisenberg ring with spin-3/2 (see text). For both panels $R_{0}=4.427 \AA$ was used as appropriate for the cyclic molecular cluster $\mathrm{Cr}_{8}$.

inspection of Eq. (15) shows that the interference terms are equivalent for $q^{\prime}$ and $N-q^{\prime}$ (and of course analogous for $q$ ). One finds

$$
\begin{aligned}
& f_{0,1}^{0}=1+\sqrt{2}\left[j_{0}\left(Q R_{1}\right)-j_{0}\left(Q R_{3}\right)\right]-j_{0}\left(Q R_{4}\right)(18 \mathrm{~b}) \\
& f_{0,2}^{0}=1-2 j_{0}\left(Q R_{2}\right)+j_{0}\left(Q R_{4}\right) \\
& f_{0,3}^{0}=1-\sqrt{2}\left[j_{0}\left(Q R_{1}\right)-j_{0}\left(Q R_{3}\right)\right]-j_{0}\left(Q R_{4}\right)(18 \mathrm{~d})
\end{aligned}
$$

These functions are plotted in Fig. 1(a) with $R_{0}$ chosen as appropriate for the $\mathrm{Cr}_{8}$ cluster. To compare with experiment one additionally has to consider the quasi degeneracy of the spin levels with $q=1,7$ and $q=3,5$ because of the $|\sin (2 \pi q / N)|$ like dependence of $\epsilon(q)$ on $q$. Since this quasi degeneracy cannot be resolved experimentally, one should sum these contributions for a comparison with observable peaks. This requires a knowledge of the oscillator strengths $\tilde{\mathbf{S}}_{1}^{2}=\sum_{\alpha}\left\langle\tau q\left|S_{1 \alpha}\right| \tau^{\prime} q^{\prime}\right\rangle^{2}$ 
TABLE I: $\tilde{\mathbf{S}}_{1}^{2}=\Sigma_{\alpha}\left\langle\tau q\left|S_{1 \alpha}\right| \tau^{\prime} q^{\prime}\right\rangle^{2}$ for the possible transitions from the ground state $(S=0, q=0)$ to the $L$-band $(S=$ $\left.1, q^{\prime}=4\right)$ and to the $E$-band $\left(S=1, q^{\prime}=1,2,3,5,6,7\right)$ for an octanuclear antiferromagnetic spin-3/2 Heisenberg ring.

\begin{tabular}{ccccc}
\hline \hline $\mathrm{q}^{\prime}=$ & 1,7 & 2,6 & 3,5 & 4 \\
\hline & 0.06802 & 0.1870 & 0.4359 & 2.316 \\
\hline \hline
\end{tabular}

for each of the four contributing transitions. Due to symmetry reasons the oscillator strengths for $q^{\prime}$ and $N-q^{\prime}$ are equivalent. The dependence on $q$ is approximated by $\sqrt{1-\cos (q 2 \pi / N) / 1+\cos (q 2 \pi / N)}, \frac{47}{,}$ but for precise results the oscillator strengths should be calculated, e.g. numerically. For the possible transitions starting at the $S=0$ ground state, they are given in Table [

Combining Eqs. (16) and (18) and using Table I the $Q$-dependence of the INS intensity for the three transitions as observable for $\mathrm{Cr}_{8}$ at low temperatures can be expressed analytically and is shown in Fig. 1(b) in units of $2 / 3 N F^{2}(Q)$. These curves were found to be in excellent agreement with experiment $\underline{48}$

Since $C_{0}^{2}\left(\mathbf{R}_{n}\right)=-1 / 2$, the calculation of $f_{q q^{\prime}}^{2}(Q)$ proceeds in exactly the same way as for $f_{q q^{\prime}}^{0}(Q)$. One obtains basically Eqs. (18) multiplied by $-1 / 2$ and with $j_{0}\left(Q R_{n}\right)$ replaced by $j_{2}\left(Q R_{n}\right)$. Thus, the magnetic anisotropy in the cyclic spin clusters mixes in some $j_{2}$ contributions, but since the anisotropy in general is weak compared to the antiferromagnetic coupling, the $Q$-dependence of the INS intensity is rather well described by $f_{q q^{\prime}}^{0}(Q)$ alone.

\section{TETRANUCLEAR STARLIKE SPIN CLUSTERS}

For further illustration of the use of Eq. (13), the tetranuclear starlike clusters will be considered ${ }^{9}{ }^{9}$ In these clusters a central spin is surrounded by three spins which form an almost perfect equilateral triangle. Recently, such systems incorporating four iron(III) ions were investigated and attracted considerable interest as they were found to behave as single molecule magnets and show the still rare phenomenon of resonant quantum tunneling..$\underline{9}$ For one member of this class of compounds the INS spectra has been measured recently 30.31 The $Q$-dependence has not yet been measured reliably, 31 but can be expected to be investigated with better accuracy in the near future.

The actual symmetry of these molecules is less than threefold $^{9.30}$ They exhibit a twofold symmetry axis which passes through the central spin and one of the surrounding spins. The magnetic anisotropy, accordingly, is biaxial, but much smaller than the antiferromagnetic coupling between central spin and surrounding spins. It shall thus be neglected here. This essentially corresponds to neglecting the $L=2$ terms in Eq. (7), similar to the above discussion for the cyclic clusters. Also, the geometrical deviation from threefold symmetry is very small. It is thus a very good approximation to take the spin permutational symmetry as $D_{3}$.

The arguments to calculate the interference terms are very similar to those for the cyclic spin clusters. The new feature which arises here is that the spins divide into two classes $\gamma$. Again, the subgroup $C_{3}$ will be considered. Since there is no group element $T^{n}(n=0,1,2)$ which maps the central spin (numbered by 4 here) onto one of the surrounding spins, one obtains the two classes $\{1,2,3\}$ and $\{4\}$. It should be noted here, that one obtains exactly the same classes even for the full symmetry group $D_{3}$. The $Q$-dependence is determined by the interference terms $f_{11}^{q q^{\prime}}(Q), f_{14}^{q q^{\prime}}(Q), f_{41}^{q q^{\prime}}(Q)$, and $f_{44}^{q q^{\prime}}(Q)$ in Eq. (13a). In the present case, however, $f_{14}^{q q^{\prime}}(Q)=f_{41}^{q q^{\prime}}(Q)$ and $f_{44}^{q q^{\prime}}(Q)=F^{2}(Q) \tilde{\mathbf{S}}_{4}^{2}$. The averaged INS cross section is then

$$
\begin{array}{r}
\bar{I}_{\tau q \tau^{\prime} q^{\prime}}(Q)=\frac{2}{3} F^{2}(Q) \times \\
{\left[\tilde{\mathbf{S}}_{1} \cdot \tilde{\mathbf{S}}_{1} f_{11}^{q q^{\prime}}(Q)+\tilde{\mathbf{S}}_{1} \cdot \tilde{\mathbf{S}}_{4} f_{14}^{q q^{\prime}}(Q)+\tilde{\mathbf{S}}_{4} \cdot \tilde{\mathbf{S}}_{4}\right]}
\end{array}
$$

with

$$
\begin{aligned}
& f_{11}^{q q^{\prime}}(Q)=3+6 j_{0}\left(Q R_{12}\right) \cos \left(\frac{2 \pi}{3}\left(q-q^{\prime}\right)\right) \\
& f_{14}^{q q^{\prime}}(Q)=3 j_{0}\left(Q R_{14}\right) \delta_{q q^{\prime}} .
\end{aligned}
$$

Similar to the cyclic clusters, spin levels with $q=0$ belong to an one-dimensional IR and that with $q=1,2$ to the two-dimensional IR of $D_{3}$. The latter are thus degenerate and one should add the respective INS intensities.

In the starlike clusters, the antiferromagnetic coupling between the spin- $5 / 2$ iron(III) ions leads to a $S=5$ ground state ${ }^{9.30}$ belonging to $q=0$. The first excited states, approximately $80 \mathrm{~K}$ above the ground state, are made up of two $S=4$ levels ${ }^{9.30}$ belonging to $q=1,2$. This excitation has been observed in experiment 31 Its INS intensity is calculated as

$$
\begin{array}{r}
\bar{I}_{0,12}(Q)=\frac{2}{3} F^{2}(Q) \times \\
3\left\{2 \tilde{\mathbf{S}}_{1} \cdot \tilde{\mathbf{S}}_{1}\left[1-j_{0}\left(Q R_{12}\right)\right]+\tilde{\mathbf{S}}_{4} \cdot \tilde{\mathbf{S}}_{4}\right\}
\end{array}
$$

since, as for the cyclic clusters, the interference terms remain unchanged by $q^{\prime} \rightarrow N-q^{\prime}$. Up to a constant, the $Q$-dependence is here again determined by the spatial symmetry properties of the molecule.

Equation 20 is expected to describe the $Q$ dependence of the observed $S=5 \rightarrow 2 \times S=4$ transition well, not only because of the reasons given above, but also because the only structural element which enters is $R_{12}$. This implies a strategy to use the $Q$-dependence as a check for effects which break the $D_{3}$ spin permutational 
symmetry (the $Q \rightarrow 0$ argument is not working here because of the $Q$ independent contribution $f_{44}^{q q^{\prime}}$ ). Such effects lead in particular to a contribution proportional to $j_{0}\left(Q R_{14}\right)$ which would be absent otherwise [Eq. (20)]. Of course, $L=2$ contributions also will differ, but they remain small as the magnetic ansiotropy is much smaller than the coupling.

Transitions from the ground state to even higher lying states or within the excited states should be very difficult to observe experimentally and thus are not discussed here. But transitions within the $S=5$ ground state multiplet, corresponding to $q=0 \rightarrow q^{\prime}=0$, were observed ${ }^{30}$ The calculation of their $Q$-dependencies, however, is essentially just a repetition of the above considerations.

\section{CONCLUSIONS}

In this work, it has been shown for the two cases of the cyclic and the star like clusters how the spatial symmetry of the molecule predetermines interference terms and thereby the $Q$-dependence of the INS intensity. The symmetry elements of the spin permutational symmetry group $\mathcal{G}$ impose relations among the matrix elements at different spin sites. The number of classes $\gamma$ allowed by the spin permutational symmetry turned out to be essential: The smaller the number of classes is, the fewer spin matrix elements remain uncorrelated, and the more effective the whole procedure becomes. The two cases discussed here are in a sense extreme as the number of classes $\gamma$ produced by the symmetry operations was very small, being even one for the cyclic spin cluster.

The procedure of how to calculate the effects of the spatial symmetry has been worked out here only for the case of one-dimensional IRs. This might appear as a significant restriction, but it is sufficient for many practical cases. For instance, in the cases presented here, the full symmetry group actually had two-dimensional IRs. This has been handled by first considering a subgroup with only one-dimensional IRs, calculating the interference terms for this situation, and then, in a second step, to take into account the degeneracies imposed by the full symmetry by simply adding the corresponding intensities. In the present cases this was quite trivial as the interference terms were identical for the involved degenerate states. In the general case, one has to work out the relationship between the involved matrix elements. However, since it is entirely determined by transformation properties it can be calculated using the generalized Wigner-Eckhard theorem and the irreducible operators as appropriate for the symmetry group $\mathcal{G}, 33$

This approach worked for the cyclic and star like clusters without loss of information about the $Q$-dependence because the symmetry elements of the subgroup were complete enough to produce the minimal number of classes. That is, exactly the same class structure as for the full symmetry group was obtained. Certainly, if this does not hold, one either has to accept additional un- determined matrix elements in the analytical expression for the $Q$-dependence, or has to start right away from the general, but somewhat involved, equation Eq. (11).

It is noteworthy that the outlined strategy works for quite a number of important cases. For instance, tetranuclear clusters of the so called cubane structure are very frequent and constitutes an important class of tetranuclear compounds. Actually, a significant number of tetranuclear clusters with a different topology (e.g. grids, squares, or chains) became available only recently due to advances in inorganic chemistry. The metal centers in these complexes approximate a tetrahedron, i.e. exhibit a rather high molecular symmetry. However, the $\mathrm{C}_{4}$ subgroup is sufficient to connect all spin centers by symmetry elements resulting in only one class $\gamma$ including all spin centers. Thus, these clusters can be handled in exactly the same way as the cyclic spin clusters.

In many cases, the molecular cluster does not exhibit a high symmetry exactly, but approximates it with small distortions. The Bessel functions will be affected only slightly since deviations from the regular positions of the spin centers are smaller than the distances between the spin centers. Furthermore, the distorted structure also leads only to small changes in the parameters of the spin Hamiltonian, which typically have no effect on the energy spectrum up to first order in perturbation theory. $\underline{\underline{40}}$ As a result, the interference terms are actually rather insensitive to weak distortions from an assumed optimal symmetry. The $Q$-dependencies as calculated for the approximate symmetry are then nonetheless useful, in particular as the accuracy of experimental $Q$-dependencies is often limited.

With regard to the behavior of the INS intensity for $Q$ approaching zero, it has been found for the cyclic clusters that it drops to zero if states with different spatial spin quantum numbers are involved in the transition. Vice versa, it will approach a finite value only if the spatial spin quantum numbers coincide. This is a very general feature for clusters for which all spin centers are connected by elements of the symmetry group $\mathcal{G}$, i.e. for which only one class $\gamma$ appears. With this reasoning in mind, the different behavior of the $Q$-dependencies of the transitions observed for e.g. the dimer $\mathrm{Tb}_{2} \mathrm{Br}_{9}^{3-}$ becomes immediately transparent ${ }^{23}$ - it is a result of the inversion symmetry of the dimer. These differences for $Q \rightarrow 0$ were used in this case with advantage to confirm the assignment of observed peaks to theoretically expected transitions $\stackrel{23}{\underline{23}}$ As is clear from the discussion of the tetranuclear star like cluster, this kind of argumentation does not apply to cases in which more than one class $\gamma$ arise.

\section{Acknowledgments}

OW thanks the Deutsche Forschungsgemeinschaft for partial financial support. 
* Presently at: Department of Physics, The Ohio State University, Columbus OH43210.

E-mail: waldmann@physik.uni-erlangen.de

1 R. Sessoli et al., Nature 365, 141 (1993); L. Thomas et al., ibid. 383, 145 (1996); J. R. Friedman et al., Phys. Rev. Lett. 76, 3830 (1996); C. Sangregorio et al., ibid. 78, 4645 (1997), A. Caneschi et al., J. Magn. Magn. Mater. 177-181, 1330 (1998).

2 K. L. Taft et al., J. Am. Chem. Soc. 116, 823 (1994).

3 D. Gatteschi, A. Caneschi, L. Pardi, and R. Sessoli, Science 265, 1054 (1994).

4 A. Chiolero and D. Loss, Phys. Rev. Lett. 80, 169 (1988).

5 O. Waldmann, J. Hassmann, P. Müller, G. S. Hannan, D. Volkmer, U. S. Schubert, and J.-M. Lehn, Phys. Rev. Lett. 78, 3390 (1997).

6 O. Waldmann, L. Zhao, and L. K. Thompson, Phys. Rev. Lett. 88, 066401 (2002).

7 L. K. Thompson, O. Waldmann, and Z. Xu, in Magnetism: Molecules to Materials IV, edited by J. S. Miller and M. Drillon (Wiley-VCH, 2002), p. 173.

8 L. K. Thompson, Coord. Chem. Rev. 233-234, 193 (2003).

9 A. L. Barra et al., J. Am. Chem. Soc. 121, 5302 (1999).

10 I. Chiorescu et al., Phys. Rev. Lett. 84, 3454 (2000).

11 J. Schnack, M. Luban, and R. Modler, Europhys. Lett. 56, 863 (2001).

12 O. Kahn, Molecular Magnetism (VCH, New York, 1993).

13 A. Bencini and D. Gatteschi, Electron Paramagnetic Resonace of Exchange Coupled Clusters (Springer, Berlin, 1990).

14 A. Furrer and H. U. Güdel, J. Magn. Magn. Mater. 14, 256 (1979).

15 H. U. Güdel, in Magneto-Structural Correlations in Exchange-Coupled Systems, edited by R. D. Willet (Reidel, Amsterdam, 1985), p. 325.

16 R. Caciuffo et al., Phys. Rev. Lett. 81, 4744 (1998).

17 L. Mirebeau et al., Phys. Rev. Lett. 83, 628 (1999).

18 H. U. Güdel, A. Stebler, and A. Furrer, Inorg. Chem. 18, 1021 (1979).

19 H. U. Güdel, U. Hauser, and A. Furrer, Inorg. Chem. 18, 2730 (1979).

20 H. Andres et al., J. Am. Chem. Soc. 121, 10028 (1999).

21 A. Furrer and H. U. Güdel, Phys. Rev. Lett. 39, 657 (1977).

22 A. Stebler, H. U. Güdel, A. Furrer, and J. K. Kjems, Inorg. Chem. 21, 380 (1982).
23 A. Furrer, H. U. Güdel, H. Blank, and A. Heidemann, Phys. Rev. Lett. 62, 210 (1989).

${ }^{24}$ H. Andres et al., Chem. Phys. Lett. 289, 224 (1998).

25 J. M. Clemente-Juan et al., J. Am. Chem. Soc. 121, 10021 (1999).

${ }^{26}$ H. Andres et al., Inorg. Chem. 40, 1943 (2001).

27 J. M. Clemente-Juan et al., Chem. Eur. J. 8, 5701 (2002).

28 J. J. Borrás-Almenar, J. M. Clemente-Juan, E. Coronado, and B. S. Tsukerblat, Inorg. Chem. 38, 6081 (1999).

29 J. van Slageren et al., Chem. Eur. J. 8, 277 (2002).

30 G. Amoretti, S. Carretta, R. Caciuffo, H. Casalta, A. Cornia, M. Affronte, and D. Gatteschi, Phys. Rev. B 64, 104403 (2001).

31 G. Amoretti, R. Caciuffo, S. Carretta, A. Cornia, D. Gatteschi, J. Kulda, and E. Liviotti, Appl. Phys. A 74, S929 (2002).

32 S. Carretta et al., Phys. Rev. B 67, 094405 (2003).

33 A. Messiah, Quantum Mechanics (North-Holland Pub. Co., Amsterdam, 1965).

34 M. Tinkham, Group Theory and Quantum Mechaniscs (McGraw-Hill, New York, 1964).

35 O. Waldmann, Phys. Rev. B 61, 6138 (2000).

36 A. Cornia, M. Affronte, A. G. M. Jansen, G. L. Abbati, and D. Gatteschi, Angew. Chem. Int. Ed. 38, 2264 (1999).

37 R. W. Saalfrank, I. Bernt, E. Uller, and F. Hampel, Angew. Chem. Int. Ed. 36, 2482 (1997).

38 B. Pilawa, I. Keilhauer, R. Bofinger, D. Marinov, S. Knorr, and A. Grupp, Appl. Magn. Reson. 21, 527 (2001).

39 O. Waldmann, J. Schülein, R. Koch, P. Müller, I. Bernt, R. W. Saalfrank, H. P. Andres, H. U. Güdel, and P. Allenspach, Inorg. Chem. 38, 5879 (1999).

40 O. Waldmann, R. Koch, S. Schromm, P. Müller, I. Bernt, R. W. Saalfrank, F. Hampel, and E. Balthes, Inorg. Chem. 40, 2986 (2001).

41 M. Affronte et al., Phys. Rev. Lett. 88, 167201 (2002).

42 O. Waldmann, R. Koch, S. Schromm, P. Müller, I. Bernt, and R. W. Saalfrank, Phys. Rev. Lett. 89, 246401 (2002).

43 H. P. Andres, private communication.

44 O. Waldmann, Phys. Rev. B 65, 024424 (2002).

45 J. Schnack and M. Luban, Phys. Rev. B 63, 014418 (2001).

46 P. W. Anderson, Phys. Rev. 86, 694 (1952).

47 G. Müller, Phys. Rev. 26, 1311 (1982).

48 O. Waldmann, T. Guidi, S. Carretta, C. Mondelli, unpublished. 